\title{
Overexpression of IncRNA Dancr inhibits apoptosis and enhances autophagy to protect cardiomyocytes from endoplasmic reticulum stress injury via sponging microRNA-6324
}

\author{
JIONG LI $^{1,2^{*}}$, JING XIE $^{3 *}$, YAN-ZHEN WANG $^{2}$, YI-RONG GAN ${ }^{2}$, LING WEI $^{4}$, \\ GUAN-WANER DING ${ }^{5}$, YAN-HONG DING ${ }^{6}$ and DING-XIONG XIE ${ }^{2,7}$
}

\begin{abstract}
${ }^{1}$ Key Laboratory of Preclinical Study for New Drugs of Gansu Province, School of Basic Medical Sciences, Lanzhou University, Lanzhou, Gansu 730000; ${ }^{2}$ Gansu Cardiovascular Institute, Lanzhou, Gansu 730050;

${ }^{3}$ Department of Ultrasonic Diagnosis and ${ }^{4}$ Outpatient Department, The First People's Hospital of Lanzhou, Lanzhou, Gansu 730050; ${ }^{5}$ Medical Department, Shijiazhuang People's Medical College, Shijiazhuang, Hebei 050599;

${ }^{6}$ Anesthesiology Department, The First People's Hospital of Lanzhou, Lanzhou, Gansu 730050; ${ }^{7}$ Department of Cardiac Surgery, The Second Hospital of Lanzhou University, Lanzhou, Gansu 730030, P.R. China
\end{abstract}

Received June 24, 2020; Accepted October 19, 2020

DOI: $10.3892 / \mathrm{mmr} .2020 .11755$

\begin{abstract}
Endoplasmic reticulum stress (ERS) contributes to the pathogenesis of myocardial ischemia/reperfusion injury and myocardial infarction (MI). Long non-coding RNAs (lncRNAs) serve an important role in cardiovascular diseases, and lncRNA discrimination antagonizing non-protein coding RNA (Dancr) alleviates cardiomyocyte damage. microRNA (miR)-6324 was upregulated in MI model rats and was predicted to bind to Dancr. The present study aimed to investigate the role of Dancr in ERS-induced cardiomyocytes and the potential underlying mechanisms. Tunicamycin (Tm) was used to induce ERS. Cell viability, apoptosis and levels of associated proteins, ERS and autophagy in Dancroverexpression H9C2 cells and miR-6234 mimic-transfected H9C2 cells were assessed using Cell Counting Kit-8, TUNEL staining and western blot assay, respectively. The results suggested that Dancr expression levels and cell viability were downregulated by Tm in a concentration-dependent manner compared with the control group. Tm induced apoptosis,
\end{abstract}

Correspondence to: Dr Yan-Hong Ding, Anesthesiology Department, The First People's Hospital of Lanzhou, 1 Wujiayuan West Street, Qilihe, Lanzhou, Gansu 730050, P.R. China

E-mail: dyanhong05@163.com

Dr Ding-Xiong Xie, Gansu Cardiovascular Institute, 1 Wujiayuan West Street, Qilihe, Lanzhou, Gansu 730050, P.R. China

E-mail: xiedingxiong45@126.com

*Contributed equally

Key words: apoptosis, cardiomyocytes, endoplasmic reticulum stress, long non-coding RNA discrimination antagonizing non-protein coding RNA, myocardial infarction
ERS and autophagy, as indicated by an increased ratio of apoptotic cells, increased expression levels of Bax, cleaved (c)-caspase-3/9, glucose-regulated protein $78 \mathrm{kDa}$ (GRP78), phosphorylated (p)-inositol-requiring enzyme-1 $\alpha$ (IRE1 $\alpha$ ), spliced X-box-binding protein 1 (Xbpls), IRE1 $\alpha$, activating transcription factor (ATF)6, ATF4, Beclin 1 and microtubule associated protein 1 light chain $3 \alpha$ (LC3)II/I, and decreased expression levels of Bcl-2, unspliced Xbpl (Xbplu) and p62 in the Tm group compared with the control group. Moreover, the results indicated that compared with the Tm + overexpression (Oe)-negative control (NC) group, the Tm + Oe-Dancr group displayed decreased apoptosis, but enhanced ERS and autophagy to restore cellular homeostasis. Compared with the Tm + Oe-NC group, the Tm + Oe-Dancr group decreased the ratio of apoptotic cells, decreased expression levels of Bax, c-caspase-3/9 and Xbplu, and increased expression levels of Bcl-2, p-IRE1 $\alpha$, Xbp1s, Beclin 1 and LC3II/I. Dancr overexpression also significantly downregulated miR-6324 expression compared with Oe-NC. The Dual-luciferase reporter assay further indicated an interaction between Dancr and miR-6324. In addition, miR-6324 mimic partially reversed the effects of Dancr overexpression on Tm-induced apoptosis, ERS and autophagy. In conclusion, lncRNA Dancr overexpression protected cardiomyocytes against ERS injury via sponging miR-6324, thus inhibiting apoptosis, enhancing autophagy and restoring ER homeostasis.

\section{Introduction}

With social and economic development and the aging population, the occurrence of cardiovascular events is increasing each year (1). Among cardiovascular diseases, acute myocardial infarction (AMI), which is associated with high lethality and high disability, has become the leading cause of human mortality globally (2). AMI refers to myocardial necrosis caused by acute or continuous ischemia and hypoxia in the coronary arteries (3). Myocardial ischemia is 
a pathological state in which blood perfusion of the heart is decreased, resulting in decreased oxygen supply to the heart and abnormal energy metabolism of the myocardial cells, which cannot support the normal function of the heart (4). Persistent and acute ischemia of the heart can develop into AMI (5).

At present, the treatment strategies for AMI primarily include thrombolysis, percutaneous coronary intervention and surgical bypass surgery. The primary purpose of the therapeutic strategies is to restore the blood supply to the heart, rescue myocardial cells in the infarction area and prevent further damage caused by ischemia (6). However, the current treatment strategies have limited therapeutic effects on damaged cardiomyocytes, as cardiomyocytes in adults cannot be naturally regenerated once they are lost (7). Therefore, rescuing myocardial cells at the near-death state in the marginal area of MI by investigating the function of certain specific long noncoding RNAs (lncRNAs) or proteins is a novel research topic for treating AMI.

lncRNAs are transcripts $>200$ nucleotides in length, lacking a specific complete open reading frame and protein coding functions (8). IncRNAs function as crucial regulators via regulating gene expression at both the transcriptional and post-transcriptional levels (9). IncRNAs can regulate microRNAs (miRNAs/miRs) by serving as miRNA sponges, adsorbing the corresponding miRNA and exerting transcriptional regulation (10). A number of studies have demonstrated that lncRNAs serve important roles in the occurrence and development of human diseases, including cardiovascular diseases $(11,12)$.

lncRNA discrimination antagonizing non-protein coding RNA (Dancr) encodes human chromosome 4q12, and was first identified as an epidermal cell differentiation suppressor (13). Further investigation revealed that Dancr functions as an oncogene in various types of cancer, including hepatocellular carcinoma (14), non-small cell lung carcinoma $(15,16)$ and osteosarcoma (17). Recently, Dancr has been reported to alleviate hypoxia-induced $\mathrm{H} 9 \mathrm{c} 2$ cardiomyocyte damage by upregulating hypoxia inducible factor-1 $\alpha$ (18). In addition, Dancr decreased hypoxia- and hypoglycemia-induced damage of cerebral microvascular endothelial cells via regulating miR-33a-5p/spliced X-box-binding protein 1 (Xbp1s) (19). However, to the best of our knowledge, the effect of Dancr in myocardial ischemia and myocardial infarction has not been previously reported.

In the case of $\mathrm{MI} /$ reperfusion ( $\mathrm{R})$, endoplasmic reticulum (ER) homeostasis is destroyed, resulting in the accumulation of a large number of unfolded or misfolded proteins in the ER, triggering the unfolded protein response (UPR) and ER stress (ERS) (20). Early ERS exerts a compensatory protective effect, but excessive ERS is involved in the pathophysiological process of various cardiovascular diseases (21). Tunicamycin (Tm) is a commonly used ERS inducer (22). Previous studies have demonstrated that low doses of Tm can produce moderate ERS, which displays a certain protective effect on MI/R $(23,24)$. Moderate ERS can also induce autophagy to help the degradation of unfolded or misfolded proteins, thus alleviating ERS (25). However, excessive ERS may initiate the apoptosis reaction and inflammatory pathways, ultimately participating in the deterioration of cardiovascular disease (26). ERS in response to various adverse stimuli has been detected and has been reported to be associated with the pathogenesis of MI/R injury, myocardial hypertrophy, ischemic cardiomyopathy, diabetic cardiomyopathy and cardiac fibrosis (27). For example, miR-711 mimic could induce cardiomyocyte apoptosis after ERS-induced MI via upregulating Xbp1 (28). Xu et al (29) demonstrated that inhibition of ERS and the cell apoptosis signaling pathway could protect cardiomyocytes against MI-induced injury.

Autophagy is an important metabolic process that degrades senescent or damaged proteins and organelles into amino groups (30). Autophagy is activated in response to nutritional deficiencies or metabolic stress to maintain tissue function and homeostasis (31). Basic autophagy has been reported to be essential for maintaining normal heart function. Meanwhile, under ischemic stress, autophagy is activated to protect cardiomyocytes from ischemia or I/R injury (32). The beneficial role of autophagy in AMI in the alleviation of MI under ischemic and ischemia/ $\mathrm{R}$ injuries has been extensively reported $(33,34)$. Therefore, taking advantage of autophagy provides a potential strategy for the development of novel drugs or therapies for AMI (35).

The aforementioned studies indicated that the moderate enhancement of ERS and autophagy, together with the repression of excessive ERS and ERS-mediated apoptosis might serve as a valuable therapeutic strategy for relieving MI-induced injury. The present study aimed to investigate the roles and molecular mechanism underlying Dancr in ERS-induced cardiomyocytes to provide a novel target for the diagnosis and therapy of AMI.

\section{Materials and methods}

Cell culture and treatment. The $\mathrm{H} 9 \mathrm{c} 2$ rat embryonic cardiomyocyte cell line (American Type Culture Collection) was cultured in DMEM (Thermo Fisher Scientific, Inc.) supplemented with 10\% FBS (Gibco; Thermo Fisher Scientific, Inc.) and 100 units $/ \mathrm{ml}$ penicillin at $37^{\circ} \mathrm{C}$ in a humidified atmosphere of $5 \% \mathrm{CO}_{2}$. The medium was replaced every other day. At $70-80 \%$ confluence, cells were digested with trypsin and EDTA.

Tm (MedChemExpress) was utilized to induce ERS. H9C2 cells were treated $\mathrm{Tm}(0.1,0.5,2.5$ or $12.5 \mu \mathrm{M})$ for $6 \mathrm{~h}$ at $37^{\circ} \mathrm{C}$ and control cells were cultured in normal medium (36).

Cell transfection.Prior to transfection, the medium was replaced with serum- and antibiotic-free DMEM. To overexpress lncRNA Dancr, the recombinant full-length rat Dancr cDNA [overexpression (Oe)-Dancr] was cloned into the pcDNA3.1 vector (Thermo Fisher Scientific, Inc.). The pcDNA3.1 empty vector was used as a negative control (NC; Oe-NC). H9C2 cells (60-70\% confluence) were transfected with oe-Dancr, oe-NC, miR-6324 mimic (5'-AGUAGGCCAGACAGCAAGC-3'; Sigma-Aldrich; Merck KGaA) or mimic-NC (5'-GGUUC GUACGUACACUGUUCA-3'; Sigma-Aldrich; Merck KGaA) using Lipofectamine ${ }^{\circledR} 2000$ (Invitrogen; Thermo Fisher Scientific, Inc.). Briefly, Lipofectamine 2000 was mixed with $50 \mathrm{nM}$ plasmids or mimics, added to the cells and incubated for $6 \mathrm{~h}$ at $37^{\circ} \mathrm{C}$. Subsequently, cells were cultured in DMEM for $24 \mathrm{~h}$ and then used for subsequent experiments. Transfection 
efficiency was evaluated via reverse transcription-quantitative PCR (RT-qPCR).

Cell Counting Kit-8 (CCK-8). The CCK-8 assay was performed to assess cell viability. H9c2 cells $\left(1 \times 10^{4}\right.$ cells/ well) were cultured in 96-well plates and subjected to corresponding treatments. Subsequently, $10 \mu \mathrm{l}$ CCK- 8 reagent (Beyotime Institute of Biotechnology) was added to each well and incubated at $37^{\circ} \mathrm{C}$ for $2 \mathrm{~h}$ in the dark. The optical density of each well was measured at a wavelength of $450 \mathrm{~nm}$ using a microplate reader.

TUNEL staining. Apoptotic H9c2 cardiomyocytes were visualized by performing TUNEL staining (Nanjing KeyGen Biotech Co., Ltd.) according to the manufacturer's protocol. Briefly, H9c2 cells were cultured on cover slips. After the corresponding treatment, cells were fixed with $4 \%$ neutral buffered formalin solution for $30 \mathrm{~min}$ at room temperature. Subsequently, $50 \mu 1$ TUNEL reaction mixture was added and incubated for $1 \mathrm{~h}$ at $37^{\circ} \mathrm{C}$. The nuclei were stained with DAPI $(2 \mu \mathrm{g} / \mathrm{ml})$ at room temperature for $5 \mathrm{~min}$. After washing twice with PBS, images were captured from three fields of view using a fluorescence microscope (magnification, $\mathrm{x} 200)$.

Western blotting. Total protein was extracted from $\mathrm{H} 9 \mathrm{c} 2$ cardiomyocytes using lysis buffer (Beyotime Institute of Biotechnology) containing a protease inhibitor and phosphatase inhibitor. After being quantified using a BCA kit (Beyotime Institute of Biotechnology), equal amounts of protein $(50 \mu \mathrm{g})$ were separated via $12 \%$ SDS-PAGE and transferred to PVDF membranes. The membranes were blocked with $5 \%$ non-fat milk at $37^{\circ} \mathrm{C}$ for $2 \mathrm{~h}$. Subsequently, the membranes were incubated overnight at $4^{\circ} \mathrm{C}$ with primary antibodies targeted against: Bcl-2 (Abcam; cat. no. ab32124; 1:1,000), Bax (Abcam; cat. no. ab32503; 1:10,000), cleaved (c)-caspase-9 (Abcam; cat. no. ab32539; 1:5,000), caspase-9 (Abcam; cat. no. ab184786; 1:1,000), c-caspase-3 (Abcam; cat. no. ab32042; 1:500), caspase-3 (Abcam; cat. no. ab13847; 1:500), glucose-regulated protein $78 \mathrm{kDa}$ (GRP78; Abcam; cat. no. ab21685; 1:1,000), phosphorylated (p)-inositol-requiring enzyme-1 (IRE1) $\alpha$ (Abcam; cat. no. ab124945; 1:1,000), IRE1 $\alpha$ (Abcam; cat. no. ab37073; 1:1,000), Xbpls (ProteinTech Group, Inc.; cat. no. 24868-1AP; 1:1,000), unspliced Xbp1 (Xbplu; ProteinTech Group, Inc.; cat. no. 25997-1-AP; 1:1,000), activating transcription factor (ATF)6 (ProteinTech Group, Inc.; cat. no. 241691-AP; 1:2,000), ATF4 (ProteinTech Group, Inc.; cat. no. 10835-1-AP; 1:1,000), Beclin 1 (ProteinTech Group, Inc.; cat. no. 11306-1-AP; 1:10,000), microtubule associated protein 1 light chain $3 \alpha$ (LC3)II/I (ProteinTech Group, Inc.; cat. no. 14600-1-AP; 1:2,000), p62 (Abcam; cat. no. ab56416; 1:1,000) and GAPDH (Abcam; cat. no. ab8245; $1: 10,000)$. Following primary incubation, the membranes were incubated with horseradish peroxidase-conjugated goat anti-rabbit/mouse IgG secondary antibodies at room temperature for $2 \mathrm{~h}$. Protein bands were visualized using an enhanced chemiluminescence reagent (Thermo Fisher Scientific, Inc.) and detection system (Amersham; Cytiva). Protein expression levels were semi-quantified using
Image-Pro Plus software version 6.0 (Media Cybernetics, Inc.) with GAPDH as the loading control.

$R T-q P C R$. Total RNA was extracted from H9C2 cardiomyocytes using an RNA isolation kit (Total RNA Extraction Reagent; Vazyme Biotech Co., Ltd.). Total RNA was reverse transcribed into cDNA using a reverse transcriptase (Vazyme Biotech Co., Ltd.). Subsequently, qPCR was performed using the CFX384 Real-Time System C1000 Thermocycler (BioRad Laboratories, Inc.) and SYBR-Green ROX-mix (Vazyme Biotech Co., Ltd.). The following primers were used for qPCR: Dancr forward, 5'-CTCGGATAGAAGCGCAGGTT-3' and reverse, 5'-AGGCAAGCGGGGTCATTAAA-3'; miR-6324 forward, 5'-ATAGCTGGGGTCAAGGTGCT-3' and reverse, 5'-CTTGCTGTCTGGCCTACTGA-3'; GAPDH forward, 5'-TTGTGCAGTGCCAGCCTC-3' and reverse, 5'-GGTAACCAGGCGTCCGATAC-3'; and U6 forward, 5'-CTCGCTTCGGCAGCACA-3' and reverse, 5'-AACGCTTCACGAATTTGCGT-3'. The following thermocycling conditions were used for $\mathrm{qPCR}$ : Initial denaturation at $95^{\circ} \mathrm{C}$ for $30 \mathrm{sec}$ then 40 cycles of $95^{\circ} \mathrm{C}$ for $5 \mathrm{sec}$ and $60^{\circ} \mathrm{C}$ for $15 \mathrm{sec}$, followed by default of melt curve. miRNA and mRNA expression levels were quantified using the $2^{-\Delta \Delta \mathrm{Cq}}$ method (37) and normalized to the internal reference genes U6 and GAPDH, respectively.

Dual-luciferase reporter assay. The wild-type (WT) and mutated (MUT) Dancr fragments containing the miR-6324 binding sites were synthesized and inserted into the pmirGLO luciferase vector (Promega Corporation) to generate WT-pmirGLO and MUT-pmirGLO, respectively. For the promoter analysis, miR-6324 mimic or mimic NC were cloned into the pGL3 luciferase reporter (Promega Corporation). Cells were co-transfected with WT-pmirGLO or MUT-pmirGLO vectors and miR-6324 mimic or mimic NC. At $48 \mathrm{~h}$ posttransfection, luciferase activities were measured using a Dual-Luciferase reporter assay system (Promega Corporation) as previously described (38).

Statistical analysis. Data are presented as the mean \pm standard deviation of from at least three independent experiments. Comparisons between two groups were analyzed using the Student's t-test. Comparisons among multiple groups were analyzed using one-way ANOVA followed by Tukey's post hoc test. Statistical analyses were performed using GraphPad Prism software (version 5.0; GraphPad Software, Inc.). $\mathrm{P}<0.05$ was considered to indicate a statistically significant difference.

\section{Results}

lncRNA Dancr is downregulated upon Tm stimulation. The present study aimed to determine whether Dancr could regulate cardiomyocytes that underwent ERS. Following stimulation with different concentrations of the ERS agonist Tm $(0.1,0.5,2.5$ or $12.5 \mu \mathrm{M})$, Dancr expression levels and cell viability in $\mathrm{H} 9 \mathrm{C} 2$ cells were measured. Compared with the control group, Tm decreased Dancr mRNA expression levels (Fig. 1A) and cell viability (Fig. 1B) in a concentrationdependent manner, suggesting a modulatory effect of Dancr 

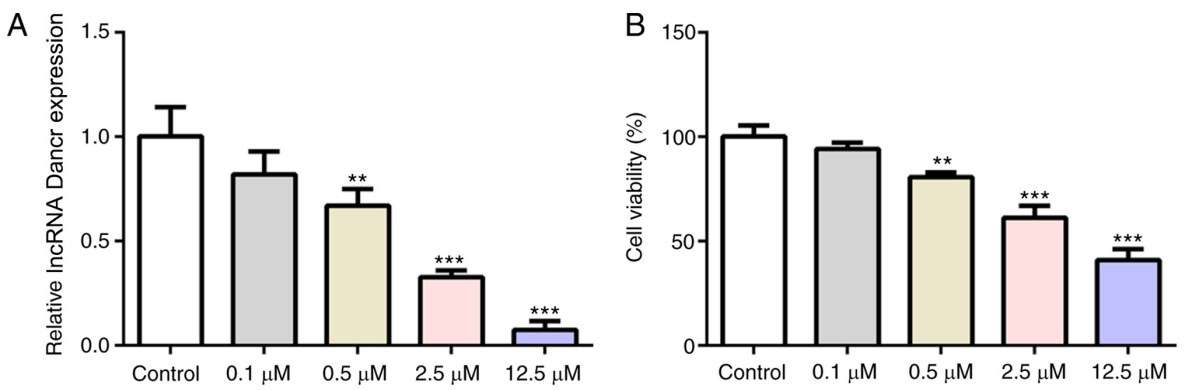

Figure 1. lncRNA Dancr is downregulated by Tm in a concentration-dependent manner. (A) Dancr mRNA expression levels and (B) cell viability in H9C2 cells treated with different concentrations of Tm. ${ }^{* *} \mathrm{P}<0.01$ and ${ }^{* * * *} \mathrm{P}<0.001$ vs. control. IncRNA, long non-coding RNA; Dancr, discrimination antagonizing non-protein coding RNA; Tm, Tunicamycin.

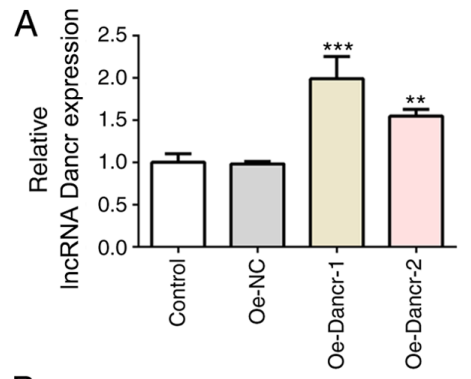

B
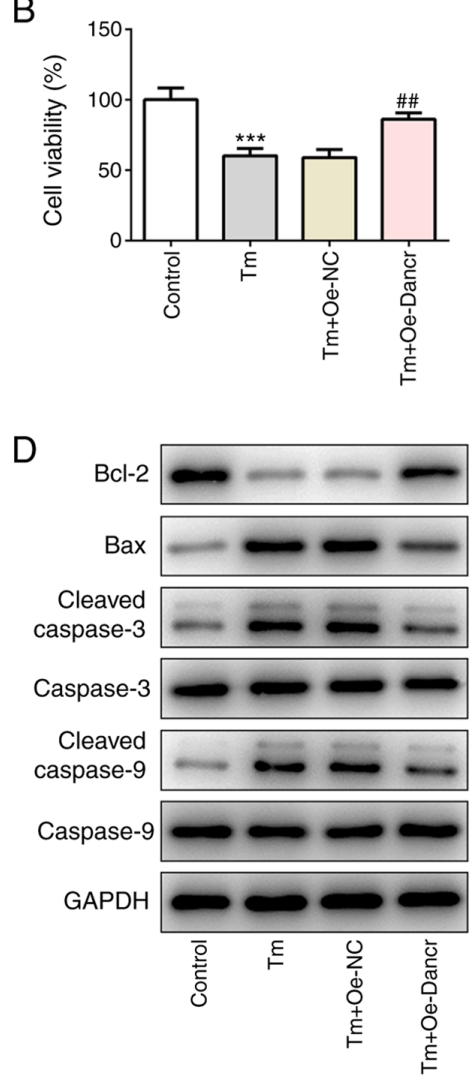

C

TUNEL
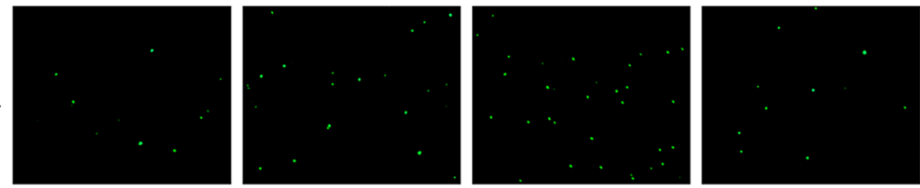

DAPI
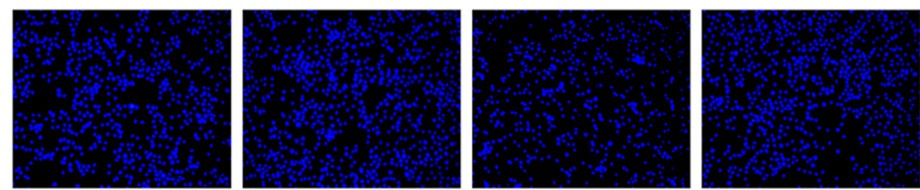

Merge

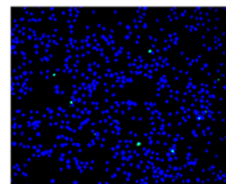

Control

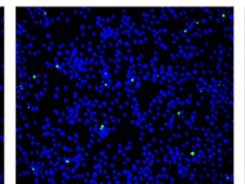

$\mathrm{Tm}$

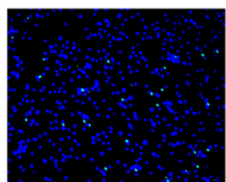

$\mathrm{Tm}+\mathrm{Oe}-\mathrm{NC}$

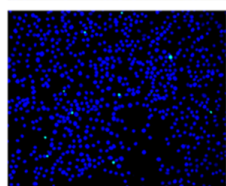

Tm+Oe-Dancr
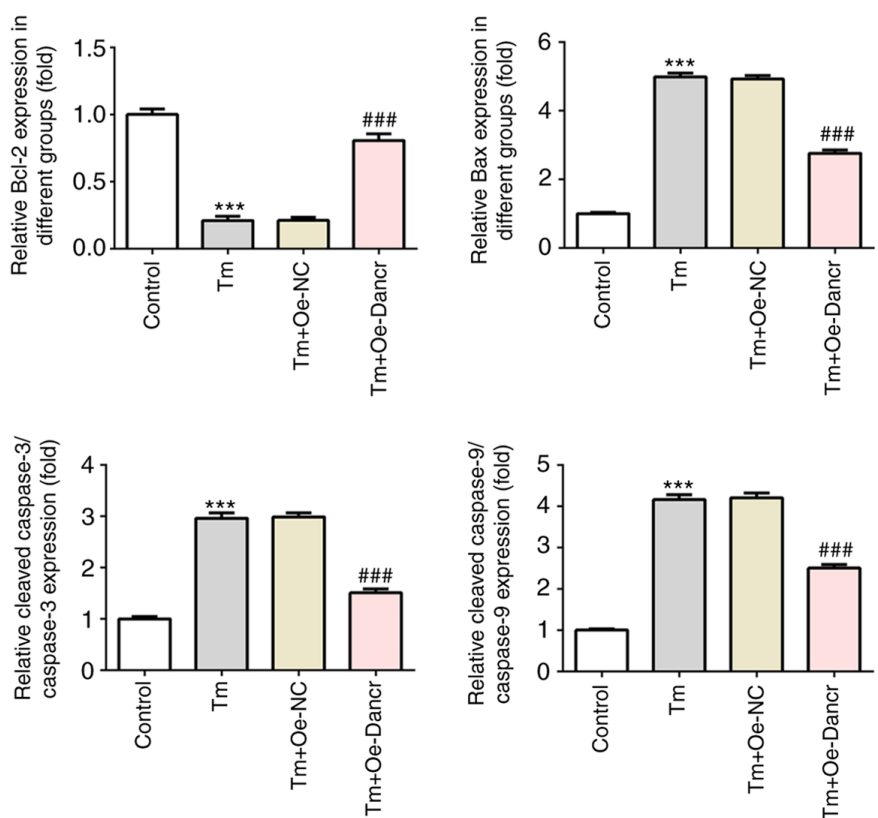

Figure 2. Effects of Dancr overexpression on cell viability and apoptosis in Tm-stimulated H9C2 cardiomyocytes. (A) Transfection efficiency of Oe-Dancr-1 and Oe-Dancr-2. ${ }^{* *} \mathrm{P}<0.01$ and ${ }^{* * *} \mathrm{P}<0.001$ vs. Oe-NC. (B) H9C2 cell viability. (C) H9C2 cell apoptosis was measured by performing TUNEL staining. Green represents apoptotic cells and blue represents nuclei (magnification, x200). (D) Western blotting was performed to measure the expression levels of apoptosisrelated proteins, including Bcl-2, Bax, cleaved-caspase-3 and cleaved caspase-9. ${ }^{* * *} \mathrm{P}<0.001$ vs. control; ${ }^{\# \#} \mathrm{P}<0.01$ and ${ }^{\# \# \# / P<0.001 ~ v s . ~ T m ~}+$ Oe-NC. Dancr, discrimination antagonizing non-protein coding RNA; Tm, Tunicamycin; Oe, overexpression; NC, negative control.

on cardiomyocyte ERS. Based on the finding that $2.5 \mu \mathrm{M}$ Tm resulted in a $>50 \%$ reduction in Dancr expression but maintained cell viability at $\geq 50 \%, 2.5 \mu \mathrm{M}$ Tm was selected for subsequent experiments (39). 

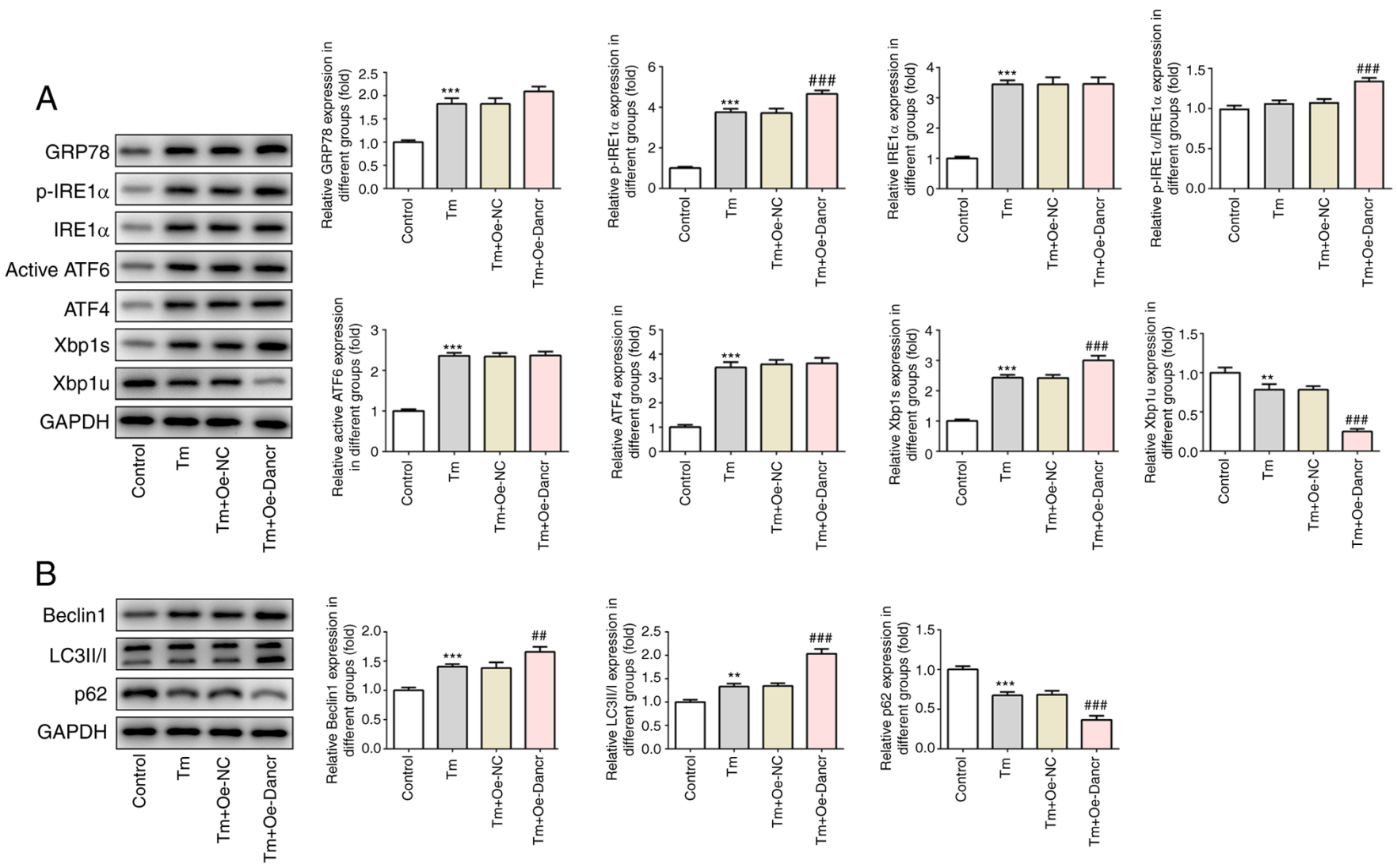

Figure 3. Effects of Dancr overexpression on ERS and autophagy. Western blotting was performed to measure the expression levels of (A) ERS-related proteins, including GRP78, p-IRE1 $\alpha$, IRE1 $\alpha$, p-IRE1 $\alpha /$ IRE1 $\alpha$, ATF6, ATF4, Xbp1s and Xbplu and (B) autophagy-related proteins, including Beclin1, LC3II/I and p62. ${ }^{* *} \mathrm{P}<0.01$ and ${ }^{* * *} \mathrm{P}<0.001$ vs. control; ${ }^{\# \#} \mathrm{P}<0.01$ and ${ }^{\# \# \#} \mathrm{P}<0.001$ vs. Tm + Oe-NC. Dancr, discrimination antagonizing non-protein coding RNA; ERS, endoplasmic reticulum stress; GRP78, glucose-regulated protein 78 kDa; p, phosphorylated; IRE1, inositol-requiring enzyme-1; ATF, activating transcription factor; Xbp1s, spliced X-box-binding protein 1; Xbplu, unspliced Xbp1; LC3, microtubule associated protein 1 light chain 3 $\alpha$; Tm, Tunicamycin; Oe, overexpression; NC, negative control.

Dancr overexpression enhances cell viability and decreases cell apoptosis in Tm-stimulated H9C2 cardiomyocytes. To determine the specific role of Dancr in ERS-induced cardiomyocyte injury, Dancr was overexpressed in H9C2 cells. The results demonstrated the successful overexpression of Dancr (Fig. 2A). Oe-Dancr-1 was selected to establish Dancroverexpression $\mathrm{H} 9 \mathrm{C} 2$ cells in subsequent experiments based on its higher efficacy compared with Oe-Dancr-2. Moreover, compared with the control group, $2.5 \mu \mathrm{M}$ Tm treatment significantly decreased cell viability, markedly increased the ratio of apoptotic cells, significantly decreased the expression levels of the antiapoptosis protein $\mathrm{Bcl}-2$, and significantly increased the expression levels of the proapoptosis proteins Bax and c-caspase-3/9 (Fig. 2B-D). However, following Tm stimulation, compared with Oe-NC-transfected cells, Oe-Dancr-transfected cells displayed significantly higher cell viability, a notably lower ratio of cell apoptosis, significantly increased expression levels of Bcl-2, and significantly decreased expression levels of Bax and c-caspase-3/9.

Dancr overexpression enhances ERS and autophagy in Tm-stimulated H9C2 cardiomyocytes. The present study analyzed the expression levels of proteins associated with ERS and autophagy. Compared with the control group, Tm treatment resulted in significantly increased expression levels of GRP78, p-IRE1 $\alpha$, Xbp1s, IRE1 $\alpha$, ATF6 and ATF4, signifi- cantly decreased expression levels of Xbplu and no significant alterations to the $\mathrm{p}-\mathrm{IRE} 1 \alpha / \mathrm{IRE} 1 \alpha$ ratio, indicating the induction of ERS (Fig. 3A). Compared with the Tm + Oe-NC group, the $\mathrm{Tm}+$ Oe-Dancr group displayed significantly enhanced p-IRE1 $\alpha, p-I R E 1 \alpha /$ IRE1 $\alpha$ and Xbp1s expression levels and significantly decreased Xbplu expression levels, but there were no significant alterations to the expression levels of IRE1 $\alpha$, ATF6 or ATF4, suggesting that Dancr selectively promoted the phosphorylation of IRE1 $\alpha$ and activated the IRE1 $\alpha$ branch in the UPR. Moreover, compared with the control group, Tm treatment induced autophagy, as evidenced by significantly increased expression levels of Beclin 1 and LC3II/I, and significantly decreased expression levels of p62 (Fig. 3B). Compared with the Tm + Oe-NC group, the Tm + Oe-Dancr group displayed increased levels of autophagy, as demonstrated by significantly increased Beclin 1 and LC3II/I expression levels, and significantly lower p62 expression levels.

miR-6324 mimic partially reverses the effects of Dancr overexpression on Tm-induced apoptosis, ERS and autophagy. miR-6324 was predicted to bind to Dancr (Fig. 4A). To verify whether miR-6324 was the target of Dancr, the present study constructed two miR-6324 mimics. The results verified the successful transfection of the miR-6324 mimics (Fig. 4B). The direct interaction between Dancr and miR-6324 was assessed by performing a Dual-luciferase reporter assay (Fig. 4C). 


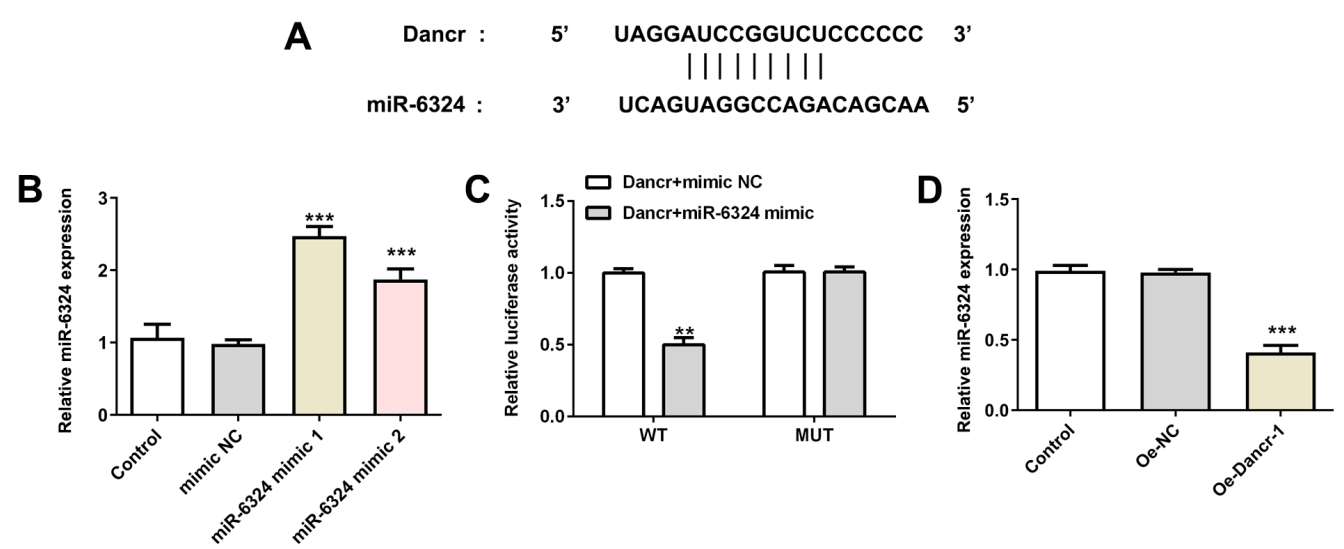

Figure 4. Relationship between Dancr and miR-6324. (A) The predicted binding sites between Dancr and miR-6324. (B) Transfection efficiency of miR-6324 mimic 1 and miR-6324 mimic 2. ${ }^{* * * *} \mathrm{P}<0.001$ vs. mimic NC. (C) A Dual-luciferase reporter assay was performed to assess the interaction between Dancr and miR-6324. ${ }^{* *} \mathrm{P}<0.01$ vs. Dancr + mimic NC. (D) miR-6324 expression levels following transfection with Oe-Dancr-1. ${ }^{* * *} \mathrm{P}<0.001$ vs. control. Dancr, discrimination antagonizing non-protein coding RNA; miR, microRNA; NC, negative control; Oe, overexpression; WT, wild-type; MUT, mutant.

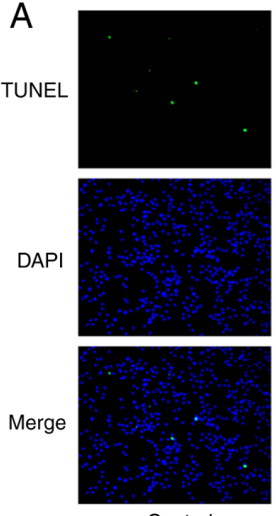

Control

B

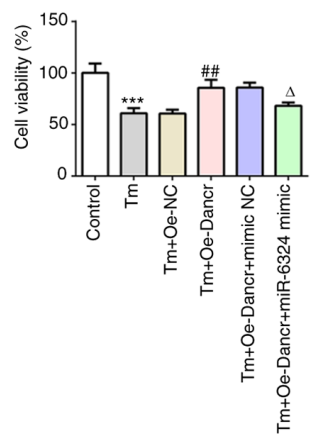

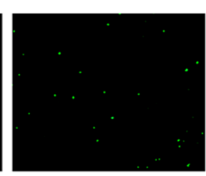
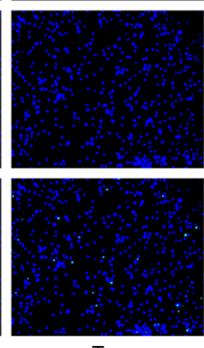

$\mathrm{Tm}$
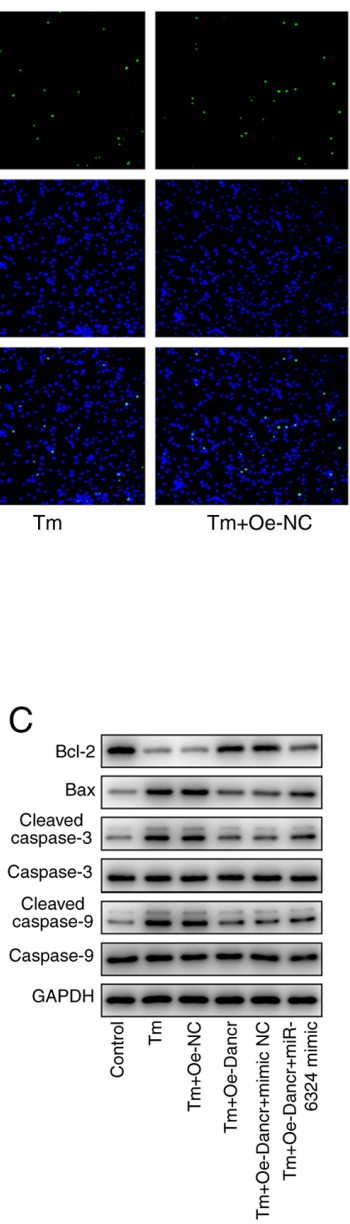

Tm+Oe-NC

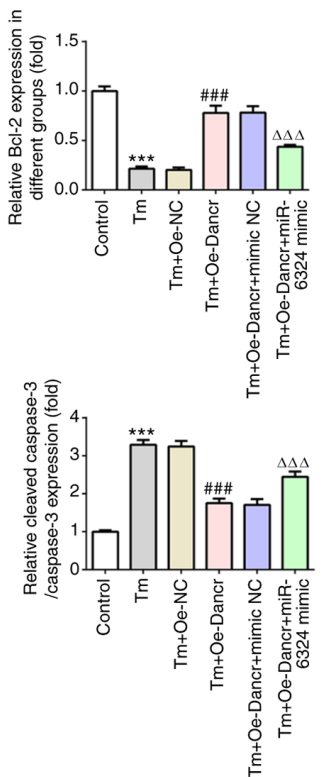

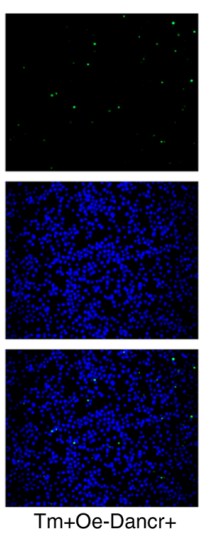

miR-6324 mimic
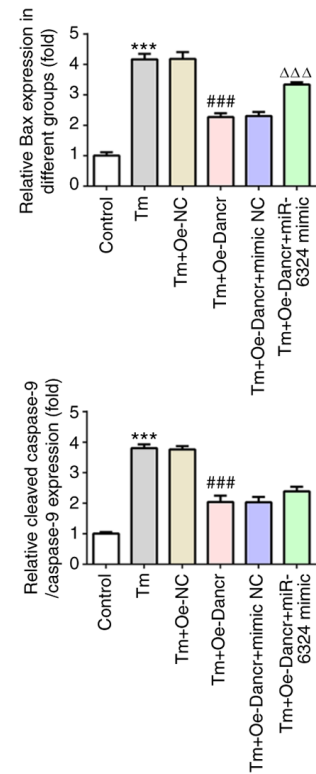

Figure 5. miR-6324 mimic reverses Dancr overexpression-mediated effects on Tm-induced apoptosis. (A) H9C2 cell apoptosis was measured by performing TUNEL staining (magnification, x200). (B) H9C2 cell viability. (C) Western blotting was performed to measure the expression levels of apoptosis-related proteins, including Bcl-2, Bax, cleaved-caspase-3 and cleaved caspase-9. ${ }^{* * *} \mathrm{P}<0.001$ vs. control; ${ }^{\# \#} \mathrm{P}<0.01$ and ${ }^{\# \# \#} \mathrm{P}<0.001$ vs. Tm + Oe-NC; ${ }^{\Delta} \mathrm{P}<0.05$ and ${ }^{\Delta \Delta \Delta} \mathrm{P}<0.001$ vs. Tm + Oe-Dancr + mimic NC. miR, microRNA; Dancr, discrimination antagonizing non-protein coding RNA; Tm, Tunicamycin; Oe, overexpression; NC, negative control.

Moreover, Dancr overexpression significantly downregulated miR-6324 expression levels compared with Oe-NC (Fig. 4D).
The results suggested that miR-6324 might participate in the actions of Dancr on ERS-induced cardiomyocyte injury. 
A

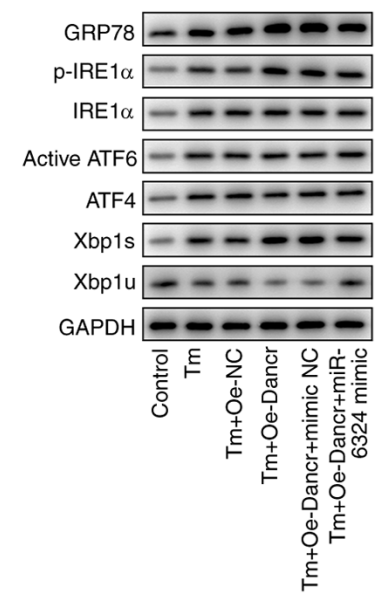

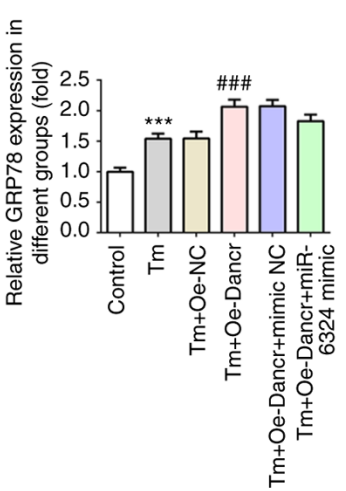

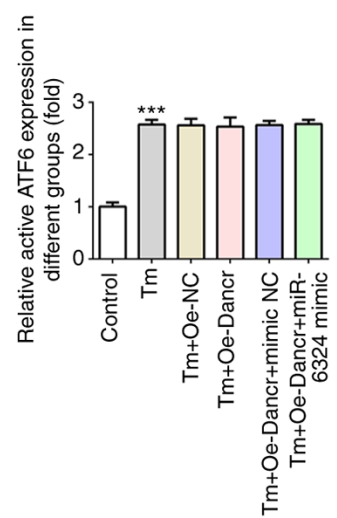

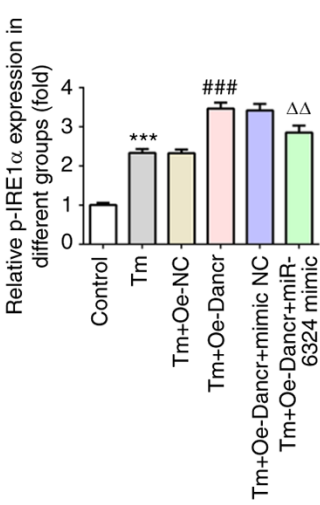
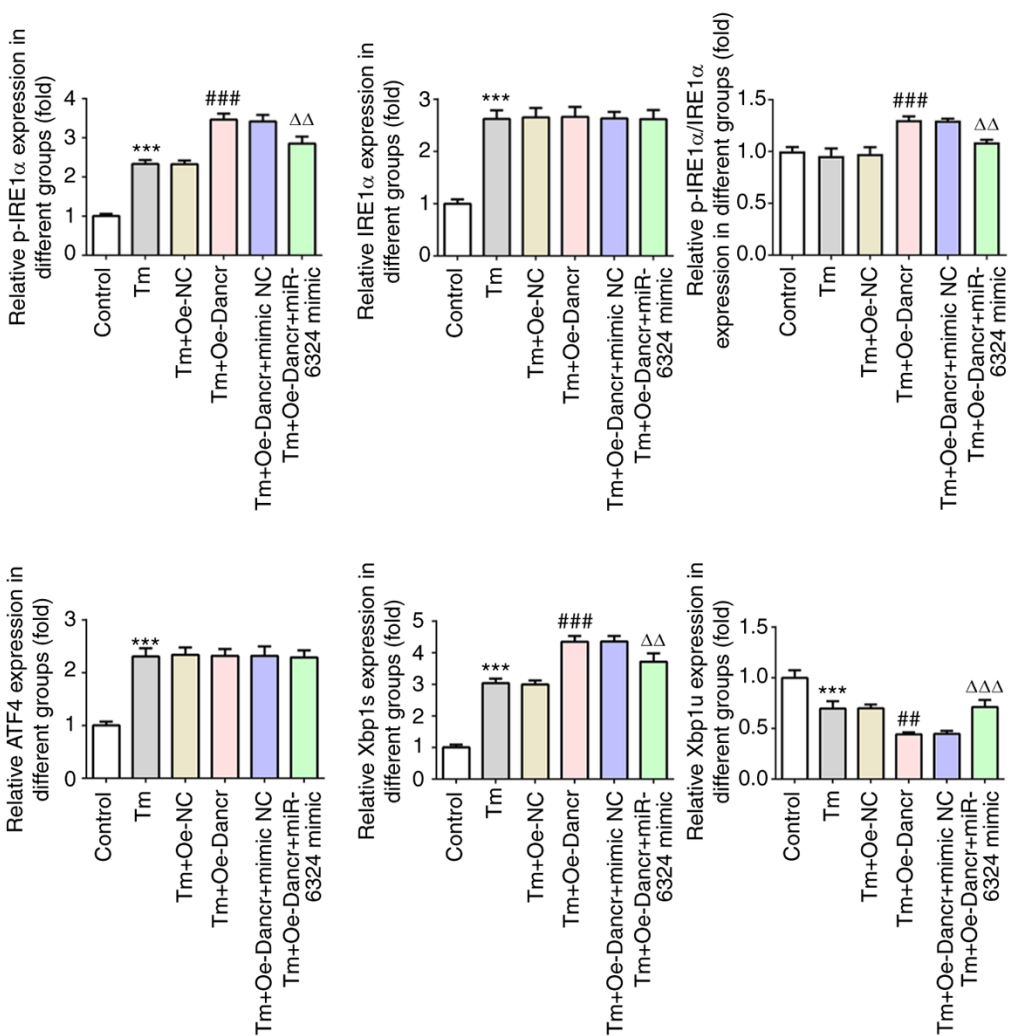

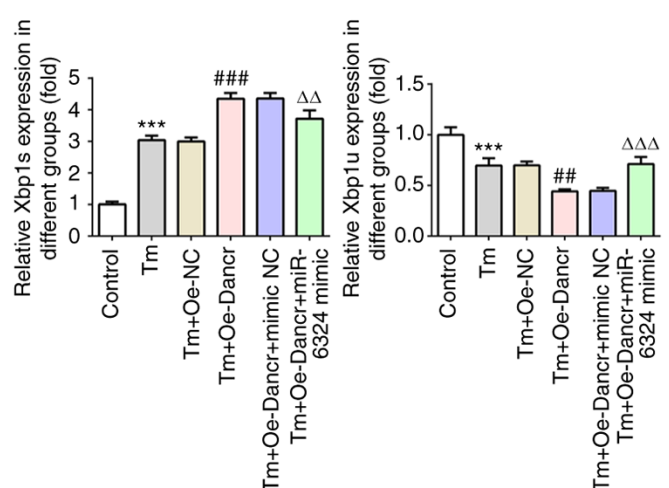

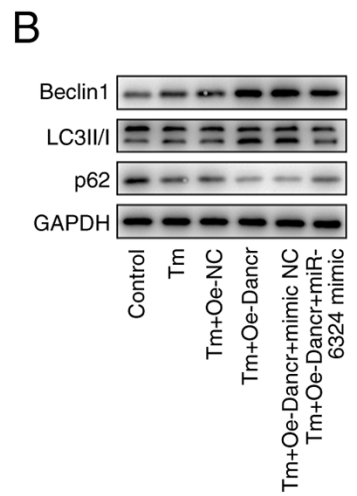
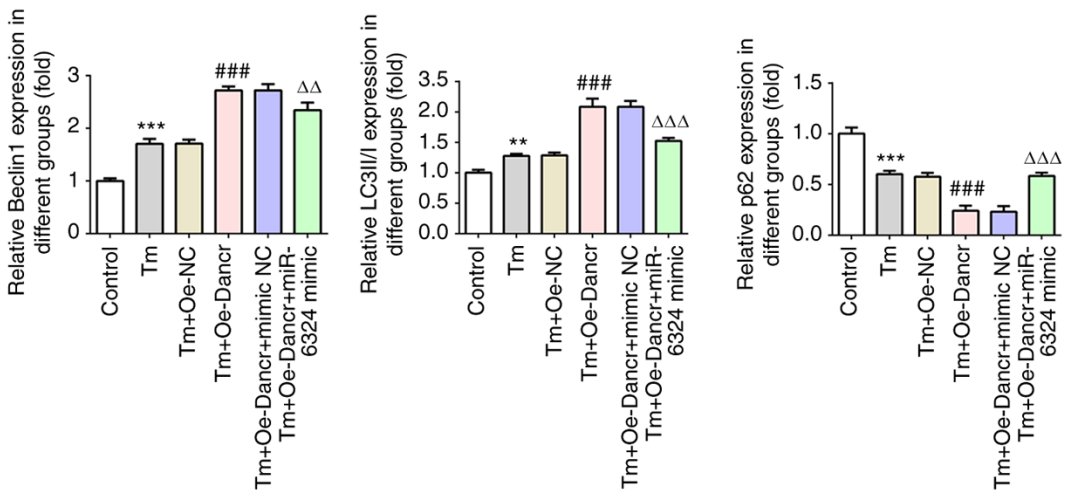

Figure 6. miR-6324 mimic reverses Dancr overexpression-mediated effects on Tm-induced ERS and autophagy. Western blotting was performed to measure the expression levels of (A) ERS-related proteins, including GRP78, p-IRE1 $\alpha$, IRE1 $\alpha$, p-IRE1 $\alpha /$ IRE1 $\alpha$, ATF6, ATF4, Xbp1s and Xbplu, (B) autophagy-related proteins, including Beclin1, LC3II/I and p62. ${ }^{* *} \mathrm{P}<0.01$ and ${ }^{* * *} \mathrm{P}<0.001$ vs. control; ${ }^{\# \#} \mathrm{P}<0.01$ and ${ }^{\# \# \#} \mathrm{P}<0.001$ vs. Tm + Oe-NC; ${ }^{\Delta \Delta} \mathrm{P}<0.01$ and ${ }^{\Delta \Delta \Delta} \mathrm{P}<0.001$ vs. $\mathrm{Tm}+$ Oe-Dancr + mimic NC. miR, microRNA; Dancr, discrimination antagonizing non-protein coding RNA; Tm, Tunicamycin; ERS, endoplasmic reticulum stress; GRP78, glucose-regulated protein $78 \mathrm{kDa}$; p, phosphorylated; IRE1, inositol-requiring enzyme-1; ATF, activating transcription factor; Xbp1s, spliced X-box-binding protein 1; Xbplu, unspliced Xbp1; LC3, microtubule associated protein 1 light chain $3 \alpha$; Oe, overexpression; NC, negative control.

To investigate the modulatory effects of miR-6324 on Dancrmediated H9C2 ERS, Tm-treated and Dancr-overexpression H9C2 cells were transfected with miR-6324 mimic or mimicNC. Compared with the Tm + Oe-Dancr + mimic NC group, the $\mathrm{Tm}+$ Oe-Dancr + miR-6324 mimic group displayed significantly decreased cell viability and markedly increased numbers of apoptotic cells (Fig. 5A and B). Furthermore, compared with the $\mathrm{Tm}+$ Oe-Dancr + mimic NC group, the $\mathrm{Tm}+$ Oe-Dancr + miR-6324 mimic group displayed significantly decreased $\mathrm{Bcl}-2$ expression levels, and significantly increased Bax and c-caspase-3/9 expression levels (Fig. 5C).

The expression levels of proteins associated with ERS and autophagy were measured (Fig. 6). Oe-Dancr-mediated upregulation of GRP78, p-IRE1 $\alpha$, p-IRE1 $\alpha /$ IRE1 $\alpha$, Xbp1s, Beclin 1 and LC3II/I expression levels was significantly reversed by miR-6324 mimic. Similarly, miR-6324 mimic partially recovered the expression levels of Xbplu and p62, which were decreased by Tm and further decreased by Dancr overexpression combined with $\mathrm{Tm}$ treatment. The aforementioned results indicated that miR-6324 mimic partially abolished Dancr overexpression-mediated effects on Tm-induced apoptosis, ERS and autophagy.

\section{Discussion}

lncRNA Dancr has been reported to protect H9c2 cardiomyocytes against hypoxia-induced damage (18). The present study indicated that Dancr protected H9C2 cells against 
ERS-induced apoptosis in vitro via the selective activation of the IRE1 $\alpha / \mathrm{XBP} 1$ signaling pathway in the UPR, which suggested that Dancr provided cytoprotection in response to ERS.

When the ER senses a wide variety of perturbations, including UPR, the adaptive process of ERS occurs to maintain ER homeostasis and mitigate or eliminate the stress (22). There are three primary ER transmembrane stress sensors that initiate the UPR, including IRE1, protein kinase RNA-like endoplasmic reticulum kinase (PERK) and ATF6, are maintained in an inactive state via binding to their luminal domains with the ER chaperone GRP78. However, in the presence of ERS, GRP78 is released from these complexes and recruited to misfolded proteins, leading to the activation of three distinct UPR branches (40). For instance, the IRE1 branch possesses endoribonuclease activity that splices mRNAs encoding Xbplu to form mature Xbp1/Xbp1s mRNA, which is then translated to a potent transcription factor that controls the genes encoding proteins that target misfolded proteins for ubiquitination and ER-associated degradation (ERAD). Meanwhile, ATF6 cooperates with IRE1 $\alpha$ for the induction of Xbp1 transcription. The proteins balance the unfolded protein/chaperone system to provide ER homeostasis. If the cell fails to recover from ERS, the UPR represses the adaptive response and triggers apoptosis. Notably, PERK stimulates the expression of the proapoptotic transcription factor $\mathrm{C} / \mathrm{EBP}$ homologous protein via the mobilization of ATF4 (41). Thus, the ERS serves a dual role, transmitting both adaptive and apoptotic signals. In the present study, compared with the control group, Tm decreased cell viability and induced cell apoptosis, which could be explained by the occurrence of excessive ERS, as evidence by increased expression levels of GRP78, p-IRE1 $\alpha$, IRE1 $\alpha$, ATF6, ATF4 and Xbp1s, and decreased expression levels of Xbplu. As previously described, if ERS is too severe or prolonged, the UPR initiates an apoptotic response (42).

Autophagy is essential for maintaining protein homeostasis and is an evolutionarily conserved protein degradation signaling pathway that removes damaged or expired proteins and organelles by chelating in autophagosomes, which subsequently undergo lysosomal degradation (43). Previous studies have demonstrated that the ERS response can activate the autophagy-lysosome signaling pathway, which serves a major role in the cardiac stress response $(44,45)$. Autophagy functions as a cellular stress signaling pathway and can assist with the degradation of proteins to recover ER homeostasis (44). In the present study, compared with the control group, Tm treatment increased the expression levels of Beclin 1 and LC3II/I, and decreased the expression levels of p62, indicating the induction of autophagy in response to ERS.

lncRNAs have been identified as novel targets for the treatment of cardiovascular diseases. For example, lncRNA CDKN2B antisense RNA 1 has been identified as the most powerful predictor of atherosclerosis $(46,47)$. Chi et al $(47)$ revealed that the IncRNA myocardial infarction associated transcript 1 led to a decrease in the expression of inflammatory factors via inhibition of the NF- $\kappa \mathrm{B}$ signaling pathway, thereby decreasing myocardial cell apoptosis and inflammatory cell infiltration to decrease AMI damage. To the best of our knowledge, the present study suggested the role of Dancr in cardiomyocyte ERS for the first time. Compared with the Tm + Oe-NC group, Dancr overexpression increased cell viability by decreasing cell apoptosis and promoting autophagy in Tm-treated $\mathrm{H} 9 \mathrm{C} 2$ cardiomyocytes. Furthermore, compared with the Tm + Oe-NC group, Dancr overexpression enhanced p-IRE1 $\alpha, p-I R E 1 \alpha /$ IRE1 $\alpha$ and Xbp1s expression levels, and decreased Xbplu expression levels, but displayed no significant effects on IRE1 $\alpha$, ATF6 and ATF4 expression levels in Tm-treated H9C2 cardiomyocytes, suggesting that Dancr selectively activated the IRE1 $\alpha$ branch in the UPR, thus promoting autophagy and ERAD, and ultimately alleviating ERS. It has been reported that enhancing autophagy could protect cardiomyocytes from ERS and apoptosis (48). However, whether the antiapoptotic effect of Dancr is dependent on the autophagy signaling pathway, the ERS signaling pathway or other mediators requires further investigation.

IncRNAs regulate the occurrence and development of human diseases, including cardiovascular diseases, primarily via sponging miRNAs (11). Our previous unpublished data demonstrated that miR-6324 was upregulated in MI model rats. In the present study, online analysis and luciferase reporter assays confirmed that miR-6324 interacted with lncRNA Dancr. Therefore, the present study hypothesized that Dancr displayed cardioprotective effects via sponging miR-6324 and inhibiting its expression. To verify the hypothesis, the present study co-transfected Tm-treated $\mathrm{H} 9 \mathrm{C} 2$ cells with Oe-Dancr and miR-6324 mimic. miR-6324 mimic partially reversed Dancr overexpression-mediated effects on cell viability, cell apoptosis, ERS and autophagy. The results indicated that miR-6324 may serve as a downstream target miRNA of Dancr to rescue cell viability, inhibit apoptosis and promote autophagy, thereby relieving cardiomyocyte ERS. However, apoptosis and autophagy are double-edged swords, thus consistent with previous studies $(20,24,25)$, the present study identified Dancr as a potential target to reduce apoptosis and ERS, while enhancing autophagy in Tm-induced H9C2 cardiomyocytes. The enhanced cell viability indicated the protective effect of Dancr, but in vivo studies are required to verify the results of the present study. Moreover, as miR-6324 mimic did not completely reverse the effects of Dancr, other downstream targets of Dancr may exist and should be investigated in future studies.

In summary, the present study provided evidence that lncRNA Dancr sponged miR-6324, selectively activated the IRE1 $\alpha / \mathrm{Xbp} 1$ signaling pathway of autophagy, repressed apoptosis and enhanced autophagy, leading to amelioration of ERS. The actions of Dancr identified in the present study suggested its potential for the treatment of cardiovascular diseases.

\section{Acknowledgements}

Not applicable.

\section{Funding}

This study was supported by the Lanzhou Talent Project for Innovation and Entrepreneurship (grant no. 2015-RC-12) and the Health Science and Technology Development Project of Lanzhou (grant no. 2019-002). 


\section{Availability of data and materials}

The datasets used and/or analyzed during the current study are available from the corresponding author on reasonable request.

\section{Authors' contributions}

YHD, DXX, JL and JX conceived and designed the study. JL, JX, YZW, YRG, LW and GWD acquired and analyzed the data. JL and JX drafted the manuscript and figures. YHD and DXX revised the manuscript for critically important intellectual content. All authors read and approved the final manuscript.

\section{Ethics approval and consent to participate}

Not applicable.

\section{Patient consent for publication}

Not applicable.

\section{Competing interests}

The authors declare that they have no competing interests.

\section{References}

1. Moran AE, Roth GA, Narula J and Mensah GA: 1990-2010 global cardiovascular disease atlas. Glob Heart 9: 3-16, 2014.

2. Mehta LS, Beckie TM, DeVon HA, Grines CL, Krumholz HM, Johnson MN, Lindley KJ, Vaccarino V, Wang TY, Watson KE, et al; American Heart Association Cardiovascular Disease in Women and Special Populations Committee of the Council on Clinical Cardiology, Council on Epidemiology and Prevention, Council on Cardiovascular and Stroke Nursing, and Council on Quality of Care and Outcomes Research: Acute Myocardial Infarction in Women: A Scientific Statement From the American Heart Association. Circulation 133: 916-947, 2016.

3. Reed GW, Rossi JE and Cannon CP: Acute myocardial infarction. Lancet 389: 197-210, 2017.

4. Tamis-Holland JE, Jneid H, Reynolds HR, Agewall S, Brilakis ES, Brown TM, Lerman A, Cushman M, Kumbhani DJ, ArslanianEngoren C, et al; American Heart Association Interventional Cardiovascular Care Committee of the Council on Clinical Cardiology; Council on Cardiovascular and Stroke Nursing; Council on Epidemiology and Prevention; and Council on Quality of Care and Outcomes Research: Contemporary Diagnosis and Management of Patients With Myocardial Infarction in the Absence of Obstructive Coronary Artery Disease: A Scientific Statement From the American Heart Association. Circulation 139: e891-e908, 2019.

5. Heusch G and Gersh BJ: The pathophysiology of acute myocardial infarction and strategies of protection beyond reperfusion: A continual challenge. Eur Heart J 38: 774-784, 2017.

6. Asaria P, Elliott P, Douglass M, Obermeyer Z, Soljak M, Majeed A and Ezzati M: Acute myocardial infarction hospital admissions and deaths in England: A national follow-back and follow-forward record-linkage study. Lancet Public Health 2: e191-e201, 2017.

7. Dall C, Khan M, Chen CA and Angelos MG: Oxygen cycling to improve survival of stem cells for myocardial repair: A review. Life Sci 153: 124-131, 2016.

8. Jarroux J, Morillon A and Pinskaya M: History, Discovery, and Classification of lncRNAs. Adv Exp Med Biol 1008: 1-46, 2017.

9. Deniz E and Erman B: Long noncoding RNA (lincRNA), a new paradigm in gene expression control. Funct Integr Genomics 17: 135-143, 2017.

10. Zhao Z, Sun W, Guo Z, Zhang J, Yu H and Liu B: Mechanisms of lncRNA/microRNA interactions in angiogenesis. Life Sci 254: $116900,2020$.
11. Sallam T, Sandhu J and Tontonoz P: Long noncoding RNA discovery in cardiovascular disease: Decoding Form to Function. Circ Res 122: 155-166, 2018.

12. Ballantyne MD, McDonald RA and Baker AH: lncRNA/ MicroRNA interactions in the vasculature. Clin Pharmacol Ther 99: 494-501, 2016.

13. Thin KZ, Liu X, Feng $X$, Raveendran $S$ and Tu JC: LncRNA-DANCR: A valuable cancer related long non-coding RNA for human cancers. Pathol Res Pract 214: 801-805, 2018.

14. Yuan SX, Wang J, Yang F, Tao QF, Zhang J, Wang LL, Yang Y, Liu H, Wang ZG, Xu QG, et al: Long noncoding RNA DANCR increases stemness features of hepatocellular carcinoma by derepression of CTNNB1. Hepatology 63: 499-511, 2016.

15. Guo L, Gu J, Hou S, Liu D, Zhou M, Hua T, Zhang J, Ge Z and $\mathrm{Xu}$ J: Long non-coding RNA DANCR promotes the progression of non-small-cell lung cancer by inhibiting p21 expression. OncoTargets Ther 12: 135-146, 2018.

16. Bai Y, Zhang G, Chu H, Li P and Li J: The positive feedback loop of lncRNA DANCR/miR-138/Sox4 facilitates malignancy in non-small cell lung cancer. Am J Cancer Res 9: 270-284, 2019.

17. Wang Y,Zeng X, Wang N, Zhao W, Zhang X, Teng S, Zhang Y and Lu Z: Long noncoding RNA DANCR, working as a competitive endogenous RNA, promotes ROCK1-mediated proliferation and metastasis via decoying of miR-335-5p and miR-1972 in osteosarcoma. Mol Cancer 17: 89, 2018.

18. Qiu L, Zhao Q, Dai L, Zhu A, Xu X, Zhao S and Chen J: Long non-coding RNA DANCR alleviates hypoxia-caused H9c2 cells damage through up regulation of HIF-1 $\alpha$. Artif Cells Nanomed Biotechnol 48: 533-541, 2020.

19. Zhang M, Tang M, Wu Q, Wang Z, Chen Z, Ding H, Hu X, Lv X, Zhao S, Sun J, et al: LncRNA DANCR attenuates brain microvascular endothelial cell damage induced by oxygen-glucose deprivation through regulating of miR-33a-5p/XBP1s. Aging (Albany NY) 12: 1778-1791, 2020.

20. Zuo S, Kong D, Wang C, Liu J, Wang Y, Wan Q, Yan S, Zhang J, Tang J, Zhang Q, et al: CRTH2 promotes endoplasmic reticulum stress-induced cardiomyocyte apoptosis through m-calpain. EMBO Mol Med 10: e8237, 2018. https://doi.org/10.15252/ emmm.201708237.

21. Wang X, Xu L, Gillette TG, Jiang X and Wang ZV: The unfolded protein response in ischemic heart disease. J Mol Cell Cardiol 117: 19-25, 2018.

22. Yan M, Shu S, Guo C, Tang C and Dong Z: Endoplasmic reticulum stress in ischemic and nephrotoxic acute kidney injury. Ann Med 50: 381-390, 2018.

23. Yamamoto $\mathrm{K}$ and Ichikawa S: Tunicamycin: Chemical synthesis and biosynthesis. J Antibiot (Tokyo) 72: 924-933, 2019.

24. Wang S, Wang Z, Fan Q, Guo J, Galli G, Du G, Wang X and Xiao W: Ginkgolide K protects the heart against endoplasmic reticulum stress injury by activating the inositol-requiring enzyme $1 \alpha / \mathrm{X}$ box-binding protein-1 pathway. Br J Pharmacol 173: 2402-2418, 2016

25. Fernández A, Ordóñez R, Reiter RJ, González-Gallego J and Mauriz JL: Melatonin and endoplasmic reticulum stress: Relation to autophagy and apoptosis. J Pineal Res 59: 292-307, 2015.

26. Hu J, Huang CX, Rao PP, Cao GQ, Zhang Y, Zhou JP, Zhu LY, Liu MX and Zhang GG: MicroRNA-155 inhibition attenuates endoplasmic reticulum stress-induced cardiomyocyte apoptosis following myocardial infarction via reducing macrophage inflammation. Eur J Pharmacol 857: 172449, 2019.

27. Li X, Zhao J, Geng J, Chen F, Wei Z, Liu C, Zhang X, Li Q, Zhang J, Gao L, et al: Long non-coding RNA MEG3 knockdown attenuates endoplasmic reticulum stress-mediated apoptosis by targeting p53 following myocardial infarction. J Cell Mol Med 23: 8369-8380, 2019.

28. Zhao N, Mi L, Zhang X, Xu M, Yu H, Liu Z, Liu X, Guan G, Gao W and Wang J: Enhanced MiR-711 transcription by PPAR $\gamma$ induces endoplasmic reticulum stress-mediated apoptosis targeting calnexin in rat cardiomyocytes after myocardial infarction. J Mol Cell Cardiol 118: 36-45, 2018.

29. Xu Y, Wu L, Chen A, Xu C and Feng Q: Protective effects of olive leaf extract on acrolein-exacerbated myocardial infarction via an endoplasmic reticulum stress pathway. Int J Mol Sci 19: 493, 2018. https://doi.org/10.3390/ijms19020493.

30. Cătană CS, Atanasov AG and Berindan-Neagoe I: Natural products with anti-aging potential: Affected targets and molecular mechanisms. Biotechnol Adv 36: 1649-1656, 2018.

31. Dong Z, Chu G, Sima Y and Chen G: Djhsp90s are crucial regulators during planarian regeneration and tissue homeostasis. Biochem Biophys Res Commun 498: 723-728, 2018. 
32. Wang L, Li Y, Ning N, Wang J, Yan Z, Zhang S, Jiao X, Wang X and Liu H: Decreased autophagy induced by $\beta 1$-adrenoceptor autoantibodies contributes to cardiomyocyte apoptosis. Cell Death Dis 9: 406, 2018.

33. Aisa Z, Liao GC, Shen XL, Chen J, Li L and Jiang SB: Effect of autophagy on myocardial infarction and its mechanism. Eur Rev Med Pharmacol Sci 21: 3705-3713, 2017.

34. Kanamori H, Takemura G, Goto K, Maruyama R, Ono K, Nagao K, Tsujimoto A, Ogino A, Takeyama T, Kawaguchi T, et al: Autophagy limits acute myocardial infarction induced by permanent coronary artery occlusion. Am J Physiol Heart Circ Physiol 300: H2261-H2271, 2011.

35. Wu D, Zhang $\mathrm{K}$ and $\mathrm{Hu} \mathrm{P}$ : The role of autophagy in acute myocardial infarction. Front Pharmacol 10: 551, 2019.

36. Yu Y, Sun G, Luo Y, Wang M, Chen R, Zhang J, Ai Q, Xing N and Sun X: Cardioprotective effects of Notoginsenoside R1 against ischemia/reperfusion injuries by regulating oxidative stress- and endoplasmic reticulum stress- related signaling pathways. Sci Rep 6: 21730, 2016.

37. Livak KJ and Schmittgen TD: Analysis of relative gene expression data using real-time quantitative PCR and the 2(-Delta Delta C(T)) Method. Methods 25: 402-408, 2001.

38. Liao B, Dong S, Xu Z, Gao F, Zhang S and Liang R: LncRNA Kcnq1ot1 renders cardiomyocytes apoptosis in acute myocardial infarction model by up-regulating Tead1. Life Sci 256: 117811, 2020.

39. Lai L, Liu Y, Liu Y, Zhang N, Cao S, Zhang X and Wu D: Role of endoplasmic reticulum oxidase $1 \alpha$ in H9C2 cardiomyocytes following hypoxia/reoxygenation injury. Mol Med Rep 22: $1420-1428,2020$.

40. Walter P and Ron D: The unfolded protein response: From stress pathway to homeostatic regulation. Science 334: 1081-1086, 2011.
41. Oyadomari S and Mori M: Roles of CHOP/GADD153 in endoplasmic reticulum stress. Cell Death Differ 11: 381-389, 2004

42. Durante W: Targeting endoplasmic reticulum stress in hypoxiainduced cardiac injury. Vascul Pharmacol 83: 1-3, 2016.

43. Levy JMM, Towers CG and Thorburn A: Targeting autophagy in cancer. Nat Rev Cancer 17: 528-542, 2017

44. Lin R, Su Z, Tan X, Su Y, Chen Y, Shu X, Liang S, Wang J and Xie S: Effect of endoplasmic reticulum stress and autophagy in the regulation of post-infarct cardiac eepair. Arch Med Res 49: 576-582, 2018

45. Wiersma M, Meijering RAM, Qi XY, Zhang D, Liu T, Hoogstra-Berends F, Sibon OCM, Henning RH, Nattel S and Brundel BJJM: Endoplasmic reticulum stress is associated with autophagy and cardiomyocyte remodeling in experimental and human atrial fibrillation. J Am Heart Assoc 6: e006458, 2017. doi: 10.1161/JAHA.117.006458.

46. Holdt LM and Teupser D: Long noncoding RNA ANRIL: Lnc-ing genetic variation at the chromosome 9p21 locus to molecular mechanisms of atherosclerosis. Front Cardiovasc Med 5: 145, 2018.

47. Chi JS, Li JZ, Jia JJ, Zhang T, Liu XM and Yi L: Long non-coding RNA ANRIL in gene regulation and its duality in atherosclerosis. J Huazhong Univ Sci Technolog Med Sci 37: 816-822, 2017. doi: 10.1007/s11596-017-1812-y.

48. Chang JC, Hu WF, Lee WS, Lin JH, Ting PC, Chang HR, Shieh KR, Chen TI and Yang KT: Intermittent hypoxia induces autophagy to protect cardiomyocytes from endoplasmic reticulum stress and apoptosis. Front Physiol 10: 995, 2019.

This work is licensed under a Creative Commons Attribution-NonCommercial-NoDerivatives 4.0 International (CC BY-NC-ND 4.0) License. 